OPEN ACCESS

Edited by:

Ivan C. Moura,

INSERM 1163 CNRS-8254, France

Reviewed by:

Peter Monk

University of Sheffield Medical

School, UK

Marco Di Gioia,

Harvard Medical School and Boston

Children's Hospital, USA

${ }^{*}$ Correspondence:

David M. Mosser

dmosser@umd.edu

Specialty section:

This article was submitted to

Molecular Innate Immunity,

a section of the journal

Frontiers in Immunology

Received: 08 October 2015

Accepted: 15 February 2016

Published: 02 March 2016

Citation:

Hamidzadeh K and Mosser DM (2016) Purinergic Signaling to

Terminate TLR Responses in

Macrophages.

Front. Immunol. 7:74.

doi: $10.3389 /$ fimmu.2016.00074

\section{Purinergic Signaling to Terminate TLR Responses in Macrophages}

\author{
Kajal Hamidzadeh and David M. Mosser* \\ Department of Cell Biology and Molecular Genetics, The Maryland Pathogen Research Institute, University of Maryland, \\ College Park, MD, USA
}

Macrophages undergo profound physiological alterations when they encounter pathogen-associated molecular patterns (PAMPs). These alterations can result in the elaboration of cytokines and mediators that promote immune responses and contribute to the clearance of pathogens. These innate immune responses by myeloid cells are transient. The termination of these secretory responses is not due to the dilution of stimuli, but rather to the active downregulation of innate responses induced by the very PAMPs that initiated them. Here, we describe a purinergic autoregulatory program whereby TLR-stimulated macrophages control their activation state. In this program, TLR-stimulated macrophages undergo metabolic alterations that result in the production of ATP and its release through membrane pannexin channels. This purine nucleotide is rapidly hydrolyzed to adenosine by ectoenzymes on the macrophage surface, CD39 and CD73. Adenosine then signals through the P1 class of seven transmembrane receptors to induce a regulatory state that is characterized by the downregulation of inflammatory cytokines and the production of anti-inflammatory cytokines and growth factors. This purinergic autoregulatory system mitigates the collateral damage that would be caused by the prolonged activation of macrophages and rather allows the macrophage to maintain homeostasis. The transient activation of macrophages can be prolonged by treating macrophages with IFN- $\boldsymbol{\gamma}$. IFN- $\boldsymbol{\gamma}$-treated macrophages become less sensitive to the regulatory effects of adenosine, allowing them to sustain macrophage activation for the duration of an adaptive immune response.

Keywords: adenosine, autoimmunity, ATP, CD39, CD73, glycolysis, IFN- $\gamma$

\section{INTRODUCTION}

The central role that macrophages play in host defense has been well described and thoroughly studied. These remarkable cells can change their physiology in response to diverse environmental stimuli and become potent antimicrobial effectors. This property has been loosely called an "activation" response, and the receptors that induce this response are generally called pattern recognition receptors (PRRs) (1). More recently, the role of macrophages in mitigating inflammatory responses and contributing to the resolution of inflammation has become an area of intense study $(2,3)$. It is clear that the very cell type that could be a potent inducer of inflammatory pathology could be equally effective at reversing this pathology. The remarkable plasticity of macrophages allows this cell to be a primary mediator of homeostasis in the host $(4,5)$.

Given the remarkable differences in the physiologies of the various macrophage subsets, efforts are underway to characterize each. These characterizations would theoretically allow the 
identification of each macrophage subtype in tissue during immunity or immunopathology. However, studies to identify definitive biochemical differences between inflammatory M1 macrophages and anti-inflammatory regulatory macrophages ( $\mathrm{R}-\mathrm{M} \phi)$ have been surprisingly underdeveloped. The in vitro transcriptional responses of the so-called M1 macrophages following their exposure to a variety of TLR ligands, such as LPS or to bacteria themselves, have been reported (6-11). These studies have begun to reveal the molecules that macrophages express and the products they secrete in response to inflammatory stimuli. However, most of these studies lack a careful kinetic analysis of transcriptional responses over time. Therefore, we are left with "snap-shots" of transcriptional responses to stimuli, rather than a motion picture of the sequential transcriptional program these stimuli induce. The transcriptional responses of anti-inflammatory macrophages have also been described $(12,13)$, but again these studies generally selected only a single time to analyze macrophage transcripts. In this review, we propose that one of the difficulties in identifying definitive biochemical differences between the various macrophage cell populations is due to the transient nature of the inflammatory response of macrophages to stimuli and the compensatory regulatory changes that accompany this activation. We describe an intrinsic program where the metabolic alterations that allow for the production of inflammatory cytokines and mediators are the very alterations that give rise to the anti-inflammatory macrophage phenotype. This autoregulatory response depends on the generation of endogenous ATP by macrophages, which initiates a purinergic signaling cascade to terminate the inflammatory response to innate stimuli, resulting in a transient state of activation. Therefore, the time when one measures the transcriptional responses of macrophages to TLR stimuli is critical. We also propose that this transient state of macrophage stimulation can be prolonged and accentuated in individuals undergoing cell-mediated immune responses. This is due to a novel activity of IFN- $\gamma$, which interferes with the stimulus-dependent upregulation of adenosine receptors to block purinergic autoregulatory responses. In this way, IFN- $\gamma$ prevents the transition to a regulatory macrophage and prolongs the activation response.

\section{METABOLIC ALTERATIONS INDUCED BY THE LIGATION OF MACROPHAGE PATTERN RECOGNITION RECEPTORS}

When macrophages encounter pathogen-associated molecular patterns (PAMPs) or damage-associated molecular patterns (DAMPs) they undergo dramatic changes in their metabolism and increase their rate of aerobic glycolysis. An increase in glucose uptake by these cells results in an accumulation of lactate in M1 macrophages (12). In contrast to M1 macrophages stimulated by PAMPs, alternatively activated macrophages exposed to IL-4 or IL-13 undergo oxidative phosphorylation and electron transport. The metabolic alterations associated with M1 macrophage polarization are believed to provide short-term, immediate access to energy for innate immune functions, whereas alternative activation is thought to provide a more stable long-term metabolism to support prolonged processes associated with wound healing (14). Recent work suggests that these metabolic alterations not only accompany differential activation but also promote the polarized responses of M1 and M2 macrophages (15). The rapid alterations in metabolism that M1 macrophages undergo are thought to allow these cells to produce the cytokines and mediators associated with host defense [reviewed in Ref. (14)]. However, the increase in glycolysis by M1 macrophages results in an increase in the production of intracellular ATP by stimulated macrophages. A portion of cytosolic ATP generated by M1 macrophages is released into the extracellular milieu via pannexin-1 channels. The addition of inhibitors of either glycolysis or pannexin channels prevents ATP release from macrophages (16). This released ATP is rapidly captured and catabolized to adenosine by M1 macrophages, allowing them to transition from an inflammatory to a regulatory phenotype. Thus, the very metabolic alterations that allow M1 macrophages to promote immune responses can also prevent these cells from causing immunopathology.

\section{THE MACROPHAGE ECTOENZYMES, CD39 AND CD73}

Purinergic signaling molecules released as a result of metabolic alterations, cell death, or tissue damage can have profound effects on macrophage activation. ATP concentrations in human plasma are typically in the nanomolar range (17) but can rise to the micromolar range under inflammatory conditions (18). ATP is constitutively released from resting parenchymal cells, and the levels are intrinsic to the tissue in which the cells reside (19). ATP release from resting macrophages is quite low, but this release is substantially increased upon TLR stimulation (16). The ATP that is released by macrophages is catabolized by macrophages in a coordinated two-step process. First, ATP is hydrolyzed to AMP by the macrophage surface ectoenzyme CD39 (E-NTPDase1) in a $\mathrm{Ca}^{2+}$ - and $\mathrm{Mg}^{2+}$-dependent manner (20). AMP is then rapidly converted to adenosine by the surface Ecto5'NTase, CD73 (21). The expression of these two enzymes by macrophages can therefore determine the concentration of adenosine in the extracellular milieu immediately surrounding the macrophage.

CD39 and CD73 expression on macrophages can change depending on the macrophage activation state. In hypoxic conditions, CD39 and CD73 function is enhanced approximately sixfold (22), whereas prolonged cultivation of macrophages in complete medium appears to downregulate CD73 expression. CD39 is more highly expressed than CD73 on bone marrow derived macrophages, and this expression pattern remains relatively constant after a brief exposure of these cells to LPS. M1 macrophages have been reported to exhibit a modest decrease in the expression of both CD39 and CD73 (23), while M2 macrophages express higher levels of both (23). These results suggest that macrophages may regulate the catabolism of ATP in order to modulate their inflammatory profile. It has also been shown that CD39 is transcriptionally regulated by the cAMP/CREB second messenger pathway that can be induced following GPCR ligation $(24,25)$. This suggests a positive feedback loop where adenosine signaling upregulates CD39 to generate more adenosine. Overall, 
this work suggests that the increased expression of either/both of these cell surface enzymes can result in an amplification of the purinergic signaling pathway in macrophages.

We recently demonstrated that the addition of exogenous adenosine or ATP to macrophages can induce these cells to assume an anti-inflammatory phenotype (16) characterized by a decreased production of inflammatory cytokines and an increased expression of angiogenic factors and anti-inflammatory cytokines (12). We further demonstrated that the hydrolysis of self-released (endogenous) ATP via macrophage CD39 allows that cell to transition from an inflammatory to an immunoregulatory state (16). Macrophages derived from CD39 knockout bone marrow fail to catabolize ATP following LPS stimulation. As a result, the production of inflammatory cytokines is sustained for up to $24 \mathrm{~h}$ poststimulation, whereas wild-type macrophages stop synthesizing these cytokines after a few hours (16). Similarly, the pharmacological inhibition of CD39 activity, using the chemical inhibitor POM-1, made macrophages hyperinflammatory with increased TNF and IL-12p40 production over the course of at least $16 \mathrm{~h}$ (16). It appears that of the two ectoenzymes involved in ATP hydrolysis, CD39 has more profound effects than CD73, presumably because the conversion of AMP to adenosine can occur in the absence of CD73. It was recently demonstrated that an inhibitor of CD73 did not have a substantial role in macrophage polarization (26).

The ability of macrophages to transition to an immunoregulatory state is key in controlling pathology in an LPS model of endotoxemia. Our lab results and others have shown that CD39 on myeloid cells can decrease mortality in mouse models of sepsis $(16,27)$, and that the addition of CD39 knockout macrophages can increase mortality in this model (16). CD73 has also been demonstrated to be protective in mouse models of sepsis (28).

\section{THE RECEPTORS FOR ADENOSINE}

Macrophages respond to adenosine via four transmembrane G-protein-coupled receptors: A1R, A2aR, adenosine $2 \mathrm{~b}$ receptor (A2bR), and A3R (29). The A1 and A3 receptors are coupled to the $G_{i}$ family of proteins resulting in decreased cAMP upon stimulation. A2a receptors are high affinity $\mathrm{G} \alpha_{\mathrm{s}}$-coupled receptors that increase intracellular cAMP $(30,31)$. Similarly, the low-affinity A2b receptors can signal through $\mathrm{G} \alpha_{\mathrm{s}}$ or $\mathrm{G}_{\mathrm{q}}$ proteins, also resulting in increased cAMP $(30,32)$. When coupled to TLR stimulation, adenosine promotes the transition from an inflammatory to a regulatory macrophage (4). Adenosine is known to be immunosuppressive in macrophages as adenosine treatment leads to increased IL-10 production and decreased TNF and IL-12 production (16). We recently performed high-throughput RNA sequencing on macrophages stimulated with LPS in the presence or absence of adenosine. Macrophages stimulated with LPS in the presence of adenosine upregulated 501 transcripts relative to LPS alone and downregulated 610 transcripts. Many of the genes that were upregulated were involved in cell growth and neovascularization, whereas genes involved in inflammation were most potently downregulated by the presence of adenosine $(12,13)$. Adenosine signaling through its $\mathrm{G} \alpha_{\mathrm{s}}$-coupled receptors also leads to increased IL-10 production via posttranscriptional mechanisms (33). Adenosine is thought to inhibit the production of the inflammatory cytokine TNF by signaling through both the $\mathrm{A} 2 \mathrm{a}$ and $\mathrm{A} 2 \mathrm{~b}$ receptors (34).

Although signaling through these GPCRs modulates levels of cAMP within cells, the role of the CAMP/PKA pathway in the regulation of inflammatory cytokines by adenosine receptor signaling remains somewhat unclear. One group has indicated that the decrease in macrophage TNF production after exposure to adenosine is due to a cAMP/PKA-independent pathway, which likely involves phosphatases (35). However, others have shown that cAMP/PKA levels are inversely correlated with TNF production (36). Thus, it is possible that while cAMP itself, mainly investigated in the form of 8-bromo-cAMP, can downregulate TNF production in macrophages, adenosine may also work by additional mechanisms that have not yet been fully defined. It was shown that the A2bR interacts with NF- $\mathrm{KB}$ in order to inhibit it, and that A2bR knockout macrophages secrete less IL-10 and more IL-12 and TNF (37).

The adenosine receptors have been implicated in the pathology of a variety of diseases. These receptors are widely expressed in the brain, heart, spleen, muscle, and lung $(38,39)$. In fact, their widespread expression is one of the challenges of developing therapeutics targeting the receptors with specificity. Studies have implicated a role for both $\mathrm{A} 2 \mathrm{aR}$ and $\mathrm{A} 2 \mathrm{bR}$ in diabetes as they are involved in gluconeogenesis and glucose homeostasis as a result of increased cAMP (40-42). There is also therapeutic anti-inflammatory potential for $\mathrm{A} 2 \mathrm{aR}$ agonists in ischemia reperfusion injury (43). In atherosclerosis, A2aR and A2bR both play a role in reducing foam cell formation, which is a feature of this disease $(44,45)$. However, it has been shown that the lack of A2aR has a protective effect in a mouse model of hypercholesterolemia because macrophages remain inflammatory and are able to reduce atherosclerotic lesions (46). Adenosine receptors also play a role in wound healing and contribute to cytokine production by macrophages of patients with chronic obstructive pulmonary disease $(29,47)$.

\section{IFN- $\gamma$ AND THE PROLONGATION OF THE MACROPHAGE ACTIVATION RESPONSE}

Priming macrophages with IFN- $\gamma$ prior to TLR stimulation results in profound changes in their physiology and dramatically accentuates their inflammatory responses $(48,49)$. Macrophages exposed to IFN- $\gamma$ not only make greater amounts of inflammatory cytokines but also produce them for prolonged periods of time (50). In this way, IFN- $\gamma$ prolongs the activation response to promote host defense against intracellular pathogens (51). The activation of macrophages, however, comes at a cost. Inflammatory macrophages exhibiting an "IFN signature" are observed in rheumatoid arthritis, multiple sclerosis, and many other autoimmune diseases, indicating that IFN- $\gamma$ contributes to autoimmune pathogenesis by promoting chronic macrophage activation $(52,53)$. Although the ability of IFN- $\gamma$ to enhance the inflammatory potential of TLR-activated macrophages is a wellknown phenomenon, the mechanism(s) whereby IFN- $\gamma$ affects the intrinsic regulation of macrophage activation remain to be 


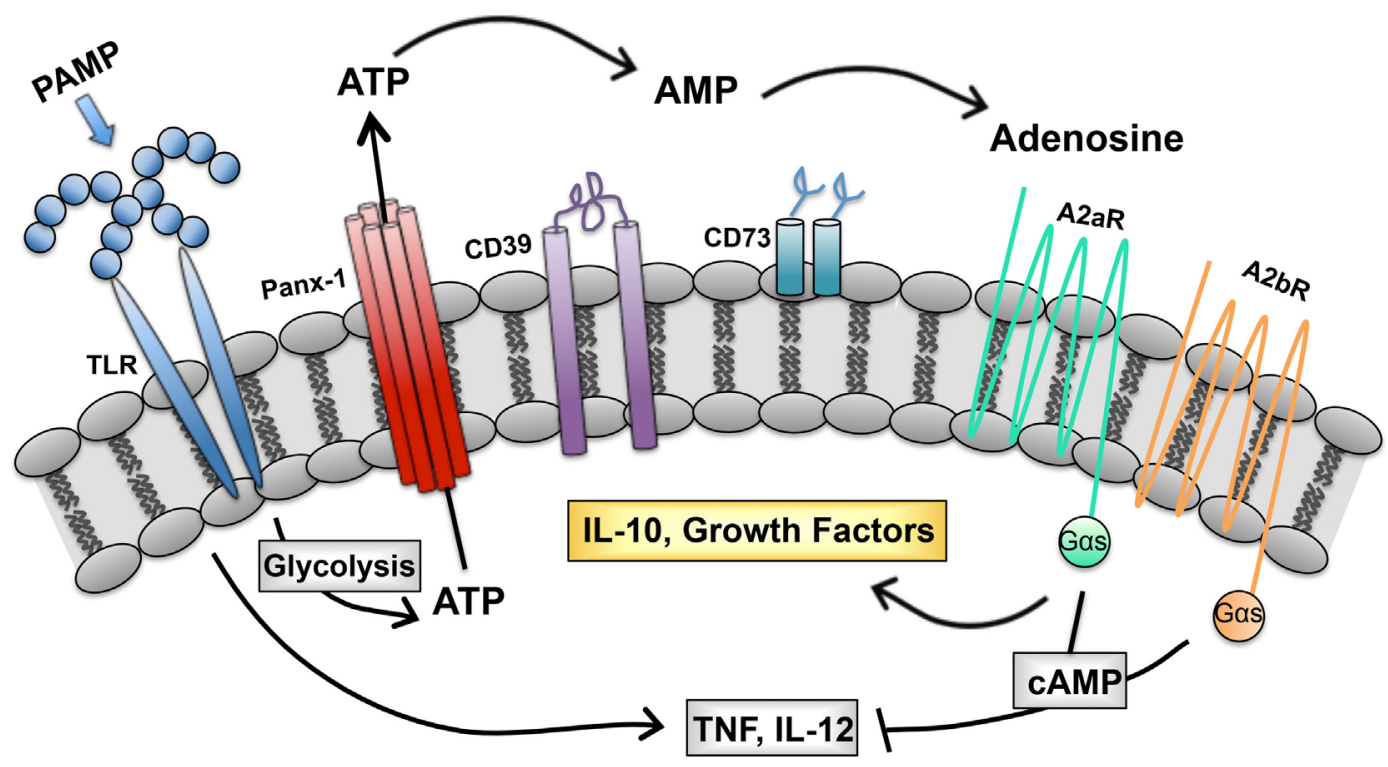

FIGURE 1 | Purinergic autoregulatory signaling in macrophages. Intracellular ATP, generated in response to pathogen-associated molecular patterns (PAMPS), is released from macrophages and converted to adenosine by the concerted action of CD39 and CD73. Adenosine signals through seven transmembrane receptors to terminate the production of inflammatory cytokines and to promote the production of IL-10 and growth factors (54).

determined. We recently identified a novel mechanism whereby IFN- $\gamma$ sustains macrophage inflammatory responses, by attenuating their sensitivity to extracellular adenosine (50).

Following TLR stimulation, macrophages dramatically upregulate their expression of receptors for adenosine. The A2bR, in particular, is upregulated more than 20 -fold in response to virtually any of the TLR ligands (50). The molecular mechanism(s) of A2bR upregulation remain to be determined, but the upregulation of adenosine receptors in response to TLR stimulation enhances macrophage sensitivity to adenosine and leads to the induction of the immunoregulatory phenotype. IFN- $\gamma$ priming of macrophages signals through STAT1 to prevent adenosine receptor induction. This decreases macrophage sensitivity to adenosine and delays the transition of macrophages to a regulatory phenotype. This prolongs the production of inflammatory cytokines such as TNF $\alpha$ and IL-12. Thus, we propose a novel mechanism whereby IFN- $\gamma$ contributes to host defense, by desensitizing macrophages to the immunoregulatory effects of adenosine. This mechanism overcomes the transient nature of TLR activation and prolongs the antimicrobial state of the classically activated macrophage.

\section{SUMMARY}

We describe a purinergic-based autoregulatory program that terminates inflammatory responses of TLR-stimulated (M1) macrophages. When macrophages are so stimulated, they undergo metabolic alterations that result in ATP generation and release through pannexin channels. Extracellular ATP is rapidly hydrolyzed to adenosine by CD39 and CD73, two ectoenzymes on the macrophage surface. Adenosine generated in this way binds to macrophage adenosine receptors to initiate a signaling pathway that terminates the synthesis of many inflammatory cytokines and induces the synthesis of regulatory transcripts (Figure 1). In this way, the overexuberant activation of macrophages is avoided. We propose that this program is in place to prevent the pathological consequences associated with chronic macrophage activation. We suggest that there are many ways to exploit this program to manipulate the phenotype of macrophages. The overexpression of CD39 and CD73 would be predicted to accelerate adenosine production by macrophages and promote a rapid regulatory transition. Drugs to prevent ectoenzyme downregulation may represent a new class of anti-inflammatory therapeutics. Similarly, drugs to induce adenosine receptor upregulation or prevent their downregulation may be developed as a way to interrupt macrophage-mediated inflammation. Conversely, targeting macrophage CD39 would be predicted to prevent this regulatory transition and promote the more efficient killing of intracellular pathogens by macrophages.

\section{AUTHOR CONTRIBUTIONS}

The ideas expressed in this review were developed by both authors who contributed equally to the writing of this review.

\section{FUNDING}

This work was supported in part by NIH grant R01 GM 102589. 


\section{REFERENCES}

1. Janeway CA Jr, Medzhitov R. Innate immune recognition. Annu Rev Immunol (2002) 20(1):197-216. doi:10.1146/annurev.immunol.20.083001.084359

2. Serhan $\mathrm{CN}$, Savill J. Resolution of inflammation: the beginning programs the end. Nat Immunol (2005) 6(12):1191-7. doi:10.1038/ni1276

3. Lawrence T, Willoughby DA, Gilroy DW. Anti-inflammatory lipid mediators and insights into the resolution of inflammation. Nat Rev Immunol (2002) 2(10):787-95. doi:10.1038/nri915

4. Mosser DM, Edwards JP. Exploring the full spectrum of macrophage activation. Nat Rev Immunol (2008) 8(12):958-69. doi:10.1038/nri2448

5. Wynn TA, Chawla A, Pollard JW. Macrophage biology in development, homeostasis and disease. Nature (2013) 496(7446):445-55. doi:10.1038/nature12034

6. Lawrence T, Natoli G. Transcriptional regulation of macrophage polarization: enabling diversity with identity. Nat Rev Immunol (2011) 11(11):750-61. doi: $10.1038 /$ nri3088

7. Nilsson R, Bajic VB, Suzuki H, di Bernardo D, Björkegren J, Katayama S, et al. Transcriptional network dynamics in macrophage activation. Genomics (2006) 88(2):133-42. doi:10.1016/j.ygeno.2006.03.022

8. Beyer M, Mallmann MR, Xue J, Staratschek-Jox A, Vorholt D, Krebs W, et al. High-resolution transcriptome of human macrophages. PLoS One (2012) 7(9):e45466. doi:10.1371/journal.pone.0045466

9. Price CTD, Abu Kwaik Y. The transcriptome of Legionella pneumophila-infected human monocyte-derived macrophages. PLoS One (2014) 9(12):e114914. doi:10.1371/journal.pone.0114914

10. Bent ZW, Poorey K, Brazel DM, LaBauve AE, Sinha A, Curtis DJ, et al. Transcriptomic analysis of Yersinia enterocolitica biovar 1B infecting murine macrophages reveals new mechanisms of extracellular and intracellular survival. Infect Immun (2015) 83(7):2672-85. doi:10.1128/IAI.02922-14

11. Mavromatis $\mathrm{CH}$, Bokil NJ, Totsika M, Kakkanat A, Schaale K, Cannistraci $\mathrm{CV}$, et al. The co-transcriptome of uropathogenic Escherichia coli-infected mouse macrophages reveals new insights into host-pathogen interactions. Cell Microbiol (2015) 17(5):730-46. doi:10.1111/cmi.12397

12. Fleming BD, Chandrasekaran P, Dillon LA, Dalby E, Suresh R, Sarkar A, et al. The generation of macrophages with anti-inflammatory activity in the absence of STAT6 signaling. J Leukoc Biol (2015) 98(3):395-407. doi:10.1189/ jlb.2A1114-560R

13. Xue J, Schmidt SV, Sander J, Draffehn A, Krebs W, Quester I, et al. Transcriptome-based network analysis reveals a spectrum model of human macrophage activation. Immunity (2014) 40(2):274-88. doi:10.1016/j. immuni.2014.01.006

14. Galvan-Pena S, O’Neill LAJ. Metabolic reprograming in macrophage polarization. Front Immunol (2014) 5:420. doi:10.3389/fimmu.2014.00420

15. Tannahill GM, Curtis AM, Adamik J, Palsson-McDermott EM, McGettrick AF, Goel G, et al. Succinate is an inflammatory signal that induces IL-1beta through HIF-1alpha. Nature (2013) 496(7444):238-42. doi:10.1038/ nature 11986

16. Cohen HB, Briggs KT, Marino JP, Ravid K, Robson SC, Mosser DM. TLR stimulation initiates a CD39-based autoregulatory mechanism that limits macrophage inflammatory responses. Blood (2013) 122(11):1935-45. doi:10.1182/blood-2013-04-496216

17. Gorman MW, Feigl EO, Buffington CW. Human plasma ATP concentration. Clin Chem (2007) 53(2):318-25. doi:10.1373/clinchem.2006.076364

18. Bours MJL, Swennen ELR, Di Virgilio F, Cronstein BN, Dagnelie PC. Adenosine 5'-triphosphate and adenosine as endogenous signaling molecules in immunity and inflammation. Pharmacol Ther (2006) 112(2):358-404. doi:10.1016/j.pharmthera.2005.04.013

19. Lazarowski ER, Boucher RC, Harden TK. Constitutive release of ATP and evidence for major contribution of ecto-nucleotide pyrophosphatase and nucleoside diphosphokinase to extracellular nucleotide concentrations. J Biol Chem (2000) 275(40):31061-8. doi:10.1074/jbc.M003255200

20. Kaczmarek E, Koziak K, Sévigny J, Siegel JB, Anrather J, Beaudoin AR, et al. Identification and characterization of CD39/vascular ATP diphosphohydrolase. J Biol Chem (1996) 271(51):33116-22. doi:10.1074/jbc.271.51.33116

21. Zimmermann H, Braun N. Ecto-nucleotidases - molecular structures, catalytic properties, and functional roles in the nervous system. Prog Brain Res (1999) 120:371-85. doi:10.1016/S0079-6123(08)63570-0

22. Synnestvedt K, Furuta GT, Comerford KM, Louis N, Karhausen J, Eltzschig $\mathrm{HK}$, et al. Ecto-5'-nucleotidase (CD73) regulation by hypoxia-inducible factor-1 mediates permeability changes in intestinal epithelia. J Clin Invest (2002) 110(7):993-1002. doi:10.1172/JCI15337

23. Zanin RF, Braganhol E, Bergamin LS, Campesato LF, Filho AZ, Moreira JC, et al. Differential macrophage activation alters the expression profile of NTPDase and ecto-5'-nucleotidase. PLoS One (2012) 7(2):e31205. doi:10.1371/journal.pone.0031205

24. Liao H, Hyman MC, Baek AE, Fukase K, Pinsky DJ. cAMP/CREB-mediated transcriptional regulation of ectonucleoside triphosphate diphosphohydrolase 1 (CD39) expression. J Biol Chem (2010) 285(19):14791-805. doi:10.1074/jbc. M110.116905

25. Baek AE, Kanthi Y, Sutton NR, Liao H, Pinsky DJ. Regulation of ecto-apyrase CD39 (ENTPD1) expression by phosphodiesterase III (PDE3). FASEB J (2013) 27(11):4419-28. doi:10.1096/fj.13-234625

26. Eichin D, Laurila JP, Jalkanen S, Salmi M. CD73 activity is dispensable for the polarization of M2 macrophages. PLoS One (2015) 10(8):e0134721. doi:10.1371/journal.pone.0134721

27. Csóka B, Németh ZH, Törő G, Koscsó B, Kókai E, Robson SC, et al. CD39 improves survival in microbial sepsis by attenuating systemic inflammation. FASEB J (2015) 29(1):25-36. doi:10.1096/fj.14-253567

28. Haskó G, Csóka B, Koscsó B, Chandra R, Pacher P, Thompson LF, et al. Ecto-5'-nucleotidase (CD73) decreases mortality and organ injury in sepsis. J Immunol (2011) 187(8):4256-67. doi:10.4049/jimmunol.1003379

29. Haskó G, Cronstein B. Regulation of inflammation by adenosine. Front Immunol (2013) 4:85. doi:10.3389/fimmu.2013.00085

30. Fredholm BB, Arslan G, Halldner L, Kull B, Schulte G, Wasserman W. Structure and function of adenosine receptors and their genes. Naunyn Schmiedebergs Arch Pharmacol (2000) 362(4-5):364-74. doi:10.1007/ s002100000313

31. Olah ME. Identification of A2a adenosine receptor domains involved in selective coupling to Gs. Analysis of chimeric A1/A2a adenosine receptors. J Biol Chem (1997) 272(1):337-44.

32. Pierce KD, Furlong TJ, Selbie LA, Shine J. Molecular cloning and expression of an adenosine A2b receptor from human brain. Biochem Biophys Res Commun (1992) 187(1):86-93. doi:10.1016/S0006-291X(05)81462-7

33. Németh ZH, Lutz CS, Csóka B, Deitch EA, Leibovich SJ, Gause WC, et al. Adenosine augments IL-10 production by macrophages through an A2B receptor-mediated posttranscriptional mechanism. JImmunol (2005) 175(12):8260-70. doi:10.4049/jimmunol.175.12.8260

34. Kreckler LM, Wan TC, Ge Z-D, Auchampach JA. Adenosine inhibits tumor necrosis factor-alpha release from mouse peritoneal macrophages via A2A and $\mathrm{A} 2 \mathrm{~B}$ but not the A3 adenosine receptor. J Pharmacol Exp Ther (2006) 317(1):172-80. doi:10.1124/jpet.105.096016

35. Kreckler LM, Gizewski E, Wan TC, Auchampach JA. Adenosine suppresses lipopolysaccharide-induced tumor necrosis factor-alpha production by murine macrophages through a protein kinase A- and exchange protein activated by cAMP-independent signaling pathway. J Pharmacol Exp Ther (2009) 331(3):1051-61. doi:10.1124/jpet.109.157651

36. Wall EA, Zavzavadjian JR, Chang MS, Randhawa B, Zhu X, Hsueh RC, et al. Suppression of LPS-induced TNF-alpha production in macrophages by cAMP is mediated by PKA-AKAP95-p105. Sci Signal (2009) 2(75):ra28. doi:10.1126/ scisignal.2000202

37. Sun Y, Duan Y, Eisenstein AS, Hu W, Quintana A, Lam WK, et al. A novel mechanism of control of NFKB activation and inflammation involving A2B adenosine receptors. J Cell Sci (2012) 125(19):4507-17. doi:10.1242/ jcs. 105023

38. Fredholm BB, Cunha RA, Svenningsson P. Pharmacology of adenosine A2A receptors and therapeutic applications. Curr Top Med Chem (2003) 3(4):413-26. doi:10.2174/1568026033392200

39. Aherne CM, Kewley EM, Eltzschig HK. The resurgence of A2B adenosine receptor signaling. Biochim Biophys Acta (2011) 1808(5):1329-39. doi:10.1016/j.bbamem.2010.05.016

40. Yasuda N, Inoue T, Horizoe T, Nagata K, Minami H, Kawata T, et al. Functional characterization of the adenosine receptor contributing to glycogenolysis and gluconeogenesis in rat hepatocytes. Eur J Pharmacol (2003) 459(2-3):159-66. doi:10.1016/S0014-2999(02)02832-7

41. Gonzalez-Benitez E, Guinzberg R, Diaz-Cruz A, Pina E. Regulation of glycogen metabolism in hepatocytes through adenosine receptors. Role of $\mathrm{Ca}^{2+}$ and cAMP. Eur J Pharmacol (2002) 437(3):105-11. doi:10.1016/ S0014-2999(02)01299-2 
42. Johnston-Cox H, Koupenova M, Yang D, Corkey B, Gokce N, Farb MG, et al. The A2B adenosine receptor modulates glucose homeostasis and obesity. PLoS One (2012) 7(7):e40584. doi:10.1371/journal.pone.0040584

43. Chhabra P, Linden J, Lobo P, Okusa MD, Brayman KL. The immunosuppressive role of adenosine A2A receptors in ischemia reperfusion injury and islet transplantation. Curr Diabetes Rev (2012) 8(6):419-33. doi:10.2174/157339912803529878

44. Reiss AB, Rahman MM, Chan ESL, Montesinos MC, Awadallah NW, Cronstein BN. Adenosine A2A receptor occupancy stimulates expression of proteins involved in reverse cholesterol transport and inhibits foam cell formation in macrophages. J Leukoc Biol (2004) 76(3):727-34. doi:10.1189/ jlb.0204107

45. Gessi S, Fogli E, Sacchetto V, Merighi S, Varani K, Preti D, et al. Adenosine modulates HIF-1 \{alpha\}, VEGF, IL-8, and foam cell formation in a human model of hypoxic foam cells. Arterioscler Thromb Vasc Biol (2010) 30(1):90-7. doi:10.1161/ATVBAHA.109.194902

46. Wang $\mathrm{H}$, Zhang $\mathrm{W}$, Zhu C, Bucher $\mathrm{C}$, Blazar BR, Zhang C, et al. Inactivation of the adenosine $\mathrm{A} 2 \mathrm{~A}$ receptor protects apolipoprotein E-deficient mice from atherosclerosis. Arterioscler Thromb Vasc Biol (2009) 29(7):1046-52. doi:10.1161/ATVBAHA.109.188839

47. Montesinos MC, Desai-Merchant A, Cronstein BN. Promotion of wound healing by an agonist of adenosine A receptor is dependent on tissue plasminogen activator. Inflammation (2015) 38(6):2036-41. doi:10.1007/ s10753-015-0184-3

48. Porta C, Riboldi E, Ippolito A, Sica A. Molecular and epigenetic basis of macrophage polarized activation. Semin Immunol (2015) 27(4):237-48. doi:10.1016/j.smim.2015.10.003

49. Schultze JL. Reprogramming of macrophages - new opportunities for therapeutic targeting. Curr Opin Pharmacol (2015) 26:10-5. doi:10.1016/j. coph.2015.09.007
50. Cohen HB, Ward A, Hamidzadeh K, Ravid K, Mosser DM. IFN-gamma prevents adenosine receptor (A2bR) upregulation to sustain the macrophage activation response. J Immunol (2015) 195(8):3828-37. doi:10.4049/ jimmunol.1501139

51. Scharton TM, Scott P. Natural killer cells are a source of interferon gamma that drives differentiation of $\mathrm{CD} 4+\mathrm{T}$ cell subsets and induces early resistance to Leishmania major in mice. J Exp Med (1993) 178(2):567-77. doi:10.1084/ jem.178.2.567

52. Hu X, Chakravarty SD, Ivashkiv LB. Regulation of interferon and toll-like receptor signaling during macrophage activation by opposing feedforward and feedback inhibition mechanisms. Immunol Rev (2008) 226:41-56. doi:10.1111/j.1600-065X.2008.00707.x

53. Gordon RA, Grigoriev G, Lee A, Kalliolias GD, Ivashkiv LB. The interferon signature and STAT1 expression in rheumatoid arthritis synovial fluid macrophages are induced by tumor necrosis factor alpha and counter-regulated by the synovial fluid microenvironment. Arthritis Rheum (2012) 64(10):3119-28. doi:10.1002/art.34544

54. Cohen HB, Mosser DM. Extrinsic and intrinsic control of macrophage inflammatory responses. J Leuk Biol (2013) 94:913-9. doi:10.1189/jlb0413236

Conflict of Interest Statement: DM declares partial ownership in LeukoSight, Inc., a company developing a line of anti-inflammatory therapeutics. $\mathrm{KH}$ has no conflict of interest to declare.

Copyright $\odot 2016$ Hamidzadeh and Mosser. This is an open-access article distributed under the terms of the Creative Commons Attribution License (CC BY). The use, distribution or reproduction in other forums is permitted, provided the original author(s) or licensor are credited and that the original publication in this journal is cited, in accordance with accepted academic practice. No use, distribution or reproduction is permitted which does not comply with these terms. 